\title{
CN Tower Lightning Return-Stroke Current Simulation
}

\author{
Khaled Elrodesly ${ }^{*}$ and Ali M. Hussein ${ }^{*}$
}

Electrical and Computer Engineering Department, Ryerson University, Toronto, Ontario, Canada

\begin{abstract}
Different functions have been used to model the lightning return-stroke current with the aid of direct current measurements at tall structures. In this paper, a comparison between the Pulse function and Heilder function is carried out to find out the suitability of each of these functions for simulating the lightning return-stroke current, measured at the CN Tower. An automated system for determining the $\mathrm{CN}$ Tower lightning return-stroke current waveform parameters is introduced. The curve fitting technique of this system and the estimation of the initial values of the simulation function parameters are presented. An artificial lightning signal, free of noise and reflections, is used as a reference signal for evaluating the parameter extraction system. Finally, cumulative statistics of the $\mathrm{CN}$ Tower lightning return-stroke current waveform parameters (peak, maximum rate of rise, risetime, pulse width, decay time and charge) are derived using the Pulse function parameters.
\end{abstract}

Keywords: Lightning return stroke current, Heidler function, pulse function, $\mathrm{CN}$ tower, double exponential function.

\section{INTRODUCTION}

Heidler function and its modified form have been widely used to simulate the lightning return-stroke current [1-3]. Furthermore, the derivative of Heidler function and the derivative of its modified form have been successfully used to simulate the lightning return-stroke current derivative, measured at the Toronto CN Tower [4-6].

This paper presents an in-depth investigation of Heidler function and its derivative in order to evaluate its suitability to simulate the lightning return-stroke current and its derivative, respectively, especially for tall-structures where reflections from structural discontinuities play an important role in the simulation. As part of the evaluation of Heidler function, it is compared with other relevant functions, such as the pulse function.

In the past, many functions were considered for simulating the lightning return-stroke current [7-11]. Some of these functions were found to have problems related to their discontinuities or the discontinuities of their first and second derivatives at the onset time. Such problems appear in the double exponential function and its modifications (Jones [7], Gardner [8], etc). However, the Pulse function and Heidler function do not suffer from such problems [9].

One of the objectives of this work is to simulate the $\mathrm{CN}$ Tower lightning return-stroke current and its derivative using either Heidler function or the Pulse function and their derivatives, respectively. And although this work is fundamental for the evaluation and development of tallstructure lightning return-stroke modeling [4], it is necessary for the determination of all waveform parameters of the current measured at the $\mathrm{CN}$ Tower, including the charge.

\footnotetext{
*Address correspondence to these authors at the Electrical and Computer Engineering Department, Ryerson University, 350 Victoria Street, Toronto, Ontario, M5B2K3, Canada; Tel: 416979 5052; Fax: 416979 5280;
}

E-mails:kelrodes@ryerson.ca, ahussein@ee.ryerson.ca
For $\mathrm{CN}$ Tower lightning currents with high signal-tonoise ratios and fast rates of rise, it is possible to determine some of the current wavefront parameters, namely the peak, the maximum rate of rise and the $10 \%$ to $90 \%$ risetime. However, other important current parameters, such as the pulse width, the decay time and the return-stroke charge, have been extremely difficult to determine because of current reflections resulting from the $\mathrm{CN}$ Tower's structural discontinuities, as well as the time-window limitation of the measured current derivative signal.

In this paper, all lightning return-stroke current waveform parameters are systematically obtained by, respectively, matching the simulation function or its derivative to the current or its derivative before the arrival of reflections.

The effectiveness of Heidler function or the Pulse function in simulating the $\mathrm{CN}$ Tower lightning current is evaluated by trying to fit each function to the current and determine the quality of the fit in each case.

\section{SIMULATION FUNCTIONS}

Both the Pulse and Heidler functions have the same general formulation that is based on the decoupling between a rise function $x(t)$ and a decay function $y(t)$. The rise and decay portions of a simulation function are chosen such that during the decay phase $x(t)=1$ and during the rise phase $y(t)=1$. The lightning return-stroke current simulation function is expressed in the form:

$$
I(t)=I_{\max } x(t) y(t)
$$

Since each of the rise function $x(t)$ and the decay function $y(t)$ is not actually unity during the decay phase and the rise phase, respectively, the lightning return-stroke current simulation function is modified to:

$I(t)=\frac{I_{\max }}{\eta} x(t) y(t)$ 


\subsection{The Pulse Function}

The Pulse function is another function that is used to represent the lightning return-stroke current [3]. It is mathematically defined by:

$$
\begin{aligned}
& I(t)=\frac{I_{\max }}{\eta}\left(1-e^{-\frac{t}{\tau_{1}}}\right)^{n} e^{-\frac{t}{\tau_{2}}} \\
& \eta=\left(\frac{n \tau_{2}}{\tau_{1}+n \tau_{2}}\right)^{n}\left(\frac{\tau_{1}}{\tau_{1}+n \tau_{2}}\right)^{\frac{\tau_{1}}{\tau_{2}}}
\end{aligned}
$$

While it's derivative is given by:

$$
\frac{d I(t)}{d t}=I(t)\left[\frac{n}{\tau_{1}} \frac{e^{-\frac{t}{\tau_{1}}}}{\left.1-e^{-\frac{t}{\tau_{1}}}\right)}-\frac{1}{\tau_{2}}\right]
$$

The zero crossing time of the current derivative, which corresponds to the current maximum, $t_{\max }$, is obtained from (5), when the current derivative vanishes.

$$
t_{\max }=\tau_{1} \ln \left(1+\frac{n \tau_{2}}{\tau_{1}}\right)
$$

Also, the time of occurrence of the maximum steepness $t_{m s}$ of the function is a very important current waveform parameter. By equating the second derivative of the Pulse function to zero we get:

$$
\left(\frac{n}{\tau_{1}}\right)^{2} e^{-\frac{2 t_{m s}}{\tau_{1}}}+\frac{\left(1-e^{-\frac{t_{m s}}{\tau_{1}}}\right)^{2}}{\tau_{2}^{2}}-\frac{n}{\tau_{1}^{2}} e^{-\frac{t_{m s}}{\tau_{1}}}-\frac{2 n e^{-\frac{t_{m s}}{\tau_{1}}}}{\tau_{1} \tau_{2}}\left(1-e^{-\frac{t_{m s}}{\tau_{1}}}\right)=0
$$

Equation (7) can be analytically solved to determine the time of occurrence of the current maximum steepness, $t_{m s}$. Since $t_{m s}$ takes place during the rising phase of the current where the decay portion $y(t) \approx 1$, then the Pulse function can be approximated during the rising phase as:

$$
I(t)=\frac{I_{\max }}{\eta}\left(1-e^{-\frac{t}{\tau_{1}}}\right)^{n}
$$

Using the second derivative and equating it to zero then a general form for $t_{\mathrm{ms}}$ can be obtained:

$t_{m s}=\tau_{1} \ln (n)$

\subsection{Heidler Function}

Heidler function is defined as:

$$
I(t)=\frac{I_{\max }}{\eta} \frac{\left(\frac{t}{\tau_{1}}\right)^{n}}{1+\left(\frac{t}{\tau_{1}}\right)^{n}} e^{-\frac{t}{\tau_{2}}}
$$

$$
\eta=e^{-\left(\frac{\tau_{1}}{\tau_{2}} \sqrt[n+1 \frac{n \tau_{2}}{\tau_{1}}]{1}\right)}
$$

While its derivative is:

$$
\frac{d I(t)}{d t}=I(t)\left[\frac{n}{t}-\frac{1}{\tau_{2}}-\frac{n t^{n-1}}{\left(\tau_{1}^{n}+t^{n}\right)}\right]
$$

For Heidler function, the zero crossing of the current derivative $t_{\max }$ is obtained numerically by solving the following equation:

$\frac{t_{\max }^{n+1}}{\tau_{1}^{n+1}}+\frac{t_{\max }}{\tau_{1}}=\frac{n \tau_{2}}{\tau_{1}}$

An approximate estimation of $t_{\max }$ can be determined by assuming that $n \gg 1$ and $\left(t_{\max } / \tau_{1}\right) \gg 1$. In this case, we can ignore $\left(t_{\max } / \tau\right)$ with respect to $\left(t_{\max } / \tau_{1}\right)^{n+1}$ and thus $t_{\max }$ can be put in the form:

$t_{\max }=\tau_{1} \sqrt[n+1]{\frac{n \tau_{2}}{\tau_{1}}}$

Also, the time of occurrence of the maximum steepness $t_{m s}$ of the derivative of Heidler function is obtained by equating the second derivative of Heidler function to zero producing equation (15)

$\frac{n}{t_{m s}}\left(\frac{n-1}{t_{m s}}-\frac{2}{\tau_{2}}\right)-\frac{n t_{m s}^{n-1}}{\left(\tau_{1}^{n}+\tau_{m s}^{n}\right)}\left(\frac{3 n-1}{t_{m s}}+\frac{2}{\tau_{2}}\right)+\frac{1}{\tau_{2}^{2}}=0$

The maximum steepness time $t_{m s}$ occurs during the rising phase of the current, where the decay phase function $y(t) \approx 1$, thus Heidler function, at the rising phase, can be approximated to:

$I(t)=\frac{I_{\max }}{\eta} \frac{\left(\frac{t}{\tau_{1}}\right)^{n}}{1+\left(\frac{t}{\tau_{1}}\right)^{n}}$

Equating the second derivative of Heidler function to zero, a general expression for $t_{m s}$ can be determined:

$t_{m s}^{n}=\frac{\tau_{1}^{n}(n-1)}{(n+1)}$

\section{INTIAL VALUE ESTIMATION}

In order to reach the optimal values of the simulation function parameters, the initial value estimation of these parameters need to be estimated. The impact of the initial condition on the curve fitting technique can be investigated by creating an artificial noise-free signal that resembles the measured signal before being corrupted by reflections. This artificial signal can be produced by having a signal source represented by an analytical function, such as Heidler function or the Pulse function. Setting the parameters of this artificial analytical function produces a continuous 
waveform. Then, this waveform is made to pass through a software digitizer having the same time resolution of the lightning current derivative recording system, which results in an artificial digital signal that simulates the measured signal.

Heidler function's analytical parameters are $I_{\max }, \tau_{1}, \tau_{2}$ and $n$, which are also the parameters of the Pulse function. In both cases, the initial value of $I_{\max }$ is known from the measurement as the maximum value of the current signal.

The decoupling behaviour of the Pulse function is used to separate the initial value estimation of $\tau_{2}$ from other parameters. This can be done by estimating the initial value of $\tau_{1}$ and $n$ using the approximation given by (8).

Although the value of $I_{\max }$ is known from the measurement, two points of the data are enough for calculating $\tau_{1}$ and $n$, but for better accuracy three points are used to eliminate $I_{\max }$ and $\eta$ from the equations. These three points are chosen based on the explained criterion to form:

\section{CURVE FITTING PROCESS}

The curve fitting technique used in this paper utilizes the least square method. It is an approach that minimizes the sum of the squares of the residuals between the measured data points and the calculated ones. This method is usually used when the data exhibit significant degree of error or noise; in this case the strategy is to derive a curve that represents the general trend of the data.

The lightning current derivative signal measured at the $\mathrm{CN}$ Tower exhibits different kinds of noise, including those produced by equipment-limited resolution and reflections [13]. Current reflections resulting from $\mathrm{CN}$ Tower structural discontinuities are the major cause of distortion to the lightning return-stroke current, normally injected at the tip of the Tower. For example, as shown in Fig. (1), the ground reflection produces major peaks in the current and the current derivative waveforms after $3.2 \mu \mathrm{s}$ from initial peaks. Therefore, it is necessary when evaluating the quality of the curve fitting to use some guidelines together with $R^{2}$ (Mean Square Error) in order to seek the optimal solution. These guidelines may include the time location of the current

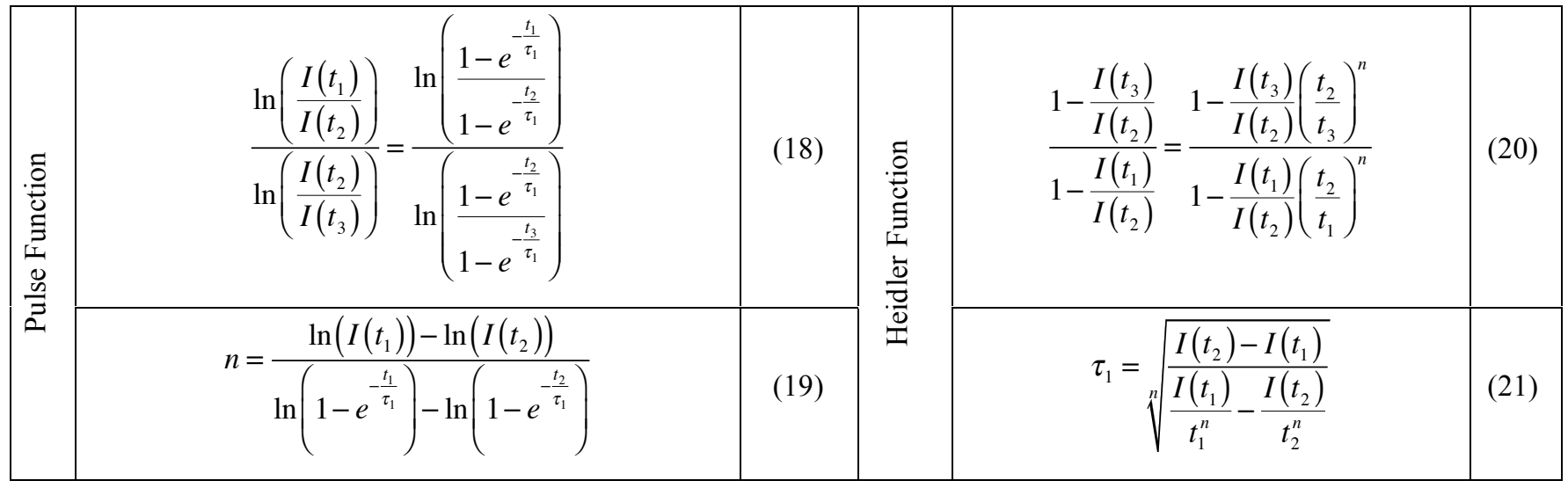

Using the same procedure for finding $\tau_{2}$ by applying the current functions on two data points where the locations of the data points satisfy the condition that their time of occurrences are greater than the time of occurrence of the maximum current so that the rising portion of the function $x(t) \approx 1$, then we divide the two equation formed using the data points to eliminate $I_{\max }$ and $\eta$.

$$
\tau_{2}=\frac{t_{5}-t_{4}}{\ln \left(\frac{I\left(t_{4}\right)}{I\left(t_{5}\right)}\right)-n \ln \left(\frac{1-e^{-\frac{t_{4}}{\tau_{1}}}}{1-e^{-\frac{t_{5}}{\tau_{1}}}}\right)}
$$

The approximation made proved to be very efficient in the determination of the initial values of the parameters. It was concluded that the simulation of the current derivative yield closer fit to the measurement, when compared with the fit resulting from simulating the current [12]. wavefront maximum steepness and the current derivative zero crossing, which corresponds to the location when the current reaches its maximum value.

Applying the explained curve fitting technique on the measured current derivative using both simulating functions, a comparison between the quality of fit using the Pulse function and Heidler function can be performed.

Using the curve fitting process, Figs. $(\mathbf{1}, \mathbf{2})$ show that the guidelines for judging the best fit to the measured signal for both functions have not been achieved. The decay portion of the simulated current is consistently lower than the measured current. The preliminary result showed that a major improvement of the simulation was necessary.

\subsection{Estimation of $\tau_{2}$}

As mentioned before, the structure of Heidler function and the Pulse function is composed of the rise portion $x(t)$ and the decay portion $y(t)$. By studying the effect of the rise term $x(t)$ on each of the simulation functions during the 

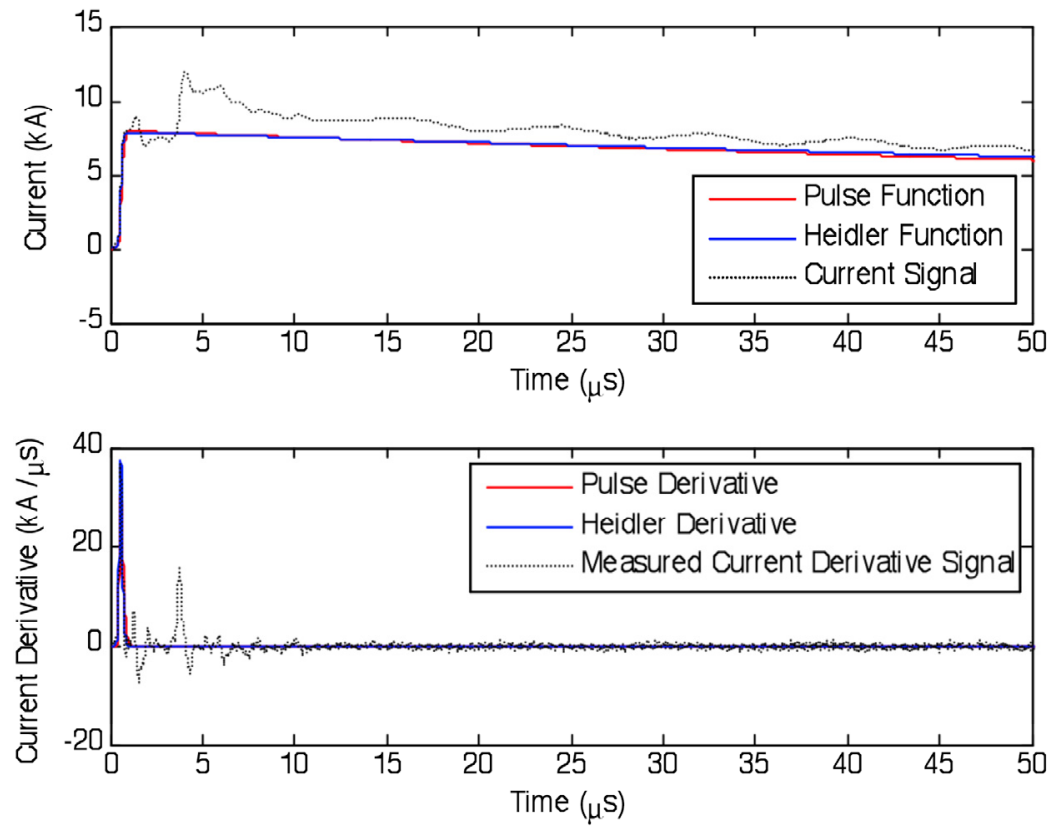

Fig. (1). Curve fitting results using the Pulse and Heidler function for simulating the measured current derivative.
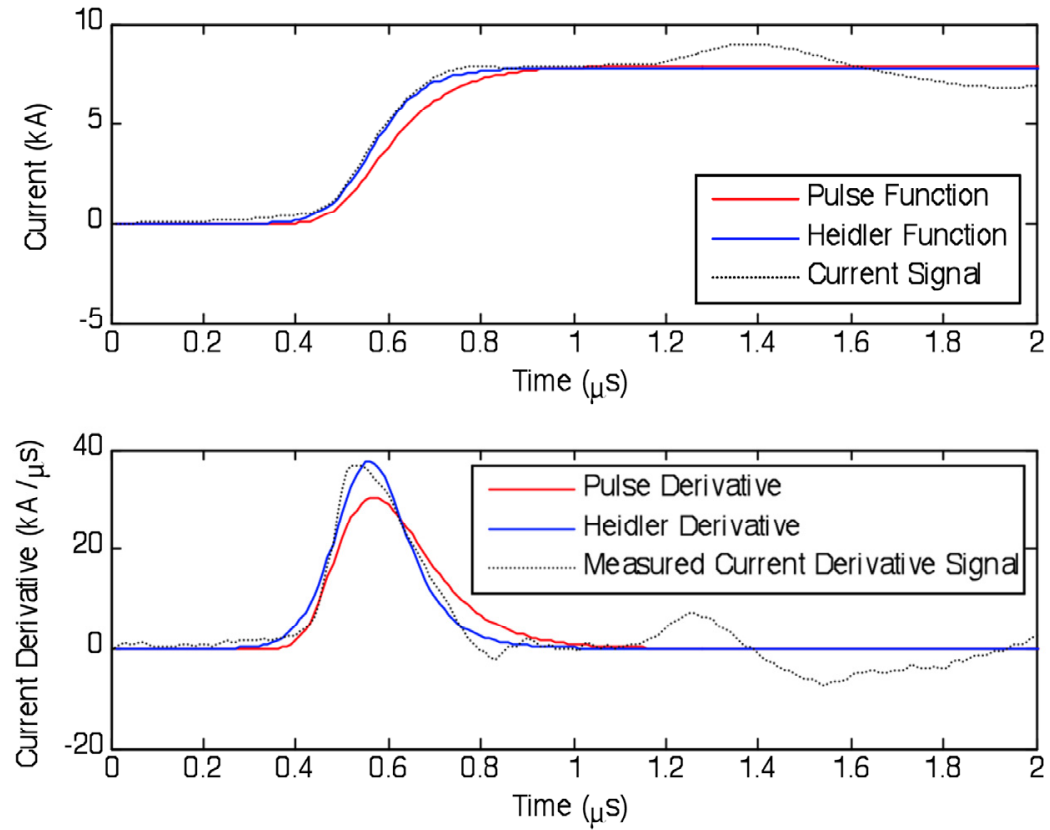

Fig. (2). Zoomed curve fitting results using the Pulse and Heidler functions for simulating the measured current derivative.

decay of the signal, when $t \gg \tau_{1}$, it is found that both functions are insensitive to the rise term where $x(t) \approx 1$ and therefore, the decay portion becomes dominant for both Heidler and the Pulse functions. In this range, both functions take the form:

$I(t)=A e^{-\frac{t}{\tau_{2}}}$

Also, its derivative will have the form:

$\frac{d I(t)}{d t}=-\frac{A}{\tau_{2}} e^{-\frac{t}{\tau_{2}}}$
In order to obtain $\tau_{2}$ separately, the measured current derivative signal and its corresponding current, obtained by numerical integration, are divided each into three timewindows. The first time-window represents the initial impulse before the arrival of reflections. The second timewindow includes the early decaying part of the waveform, which contains reflections. Finally, the third time-window represents the decay, where the waveform is less affected by reflections in comparison with its behaviour within the second time-window. The application of the curve fitting technique on the current waveform within the third timewindow, which is represented by an exponentially decaying 
function, equation (23), results in a good estimate of the decay time parameter, $\tau_{2}$.

It is important to point out that the current is used in the fitting technique to estimate $\tau_{2}$ rather the measured current derivative signal. Within the decay time-widow, the measured current derivative has a substantially lower signalto-noise ratio because of its high frequency noise, when compared with the current (see Figs. 1, 2), which leads to the failure of the fitting process.
By estimating $\tau_{2}$, the parameters of the simulation function are reduced to only three parameters, namely, $\tau_{1}$, $n$ and $I_{\max }$. Therefore, the estimation of the decay-time parameter will be always the first step in the curve fitting process of both the Pulse and Heidler functions.

The results of applying the above mentioned curve fitting technique on the current are shown in Figs. $(\mathbf{3}, \mathbf{4})$. It is noted that the rate of decay of the simulated current becomes clearly closer to that of the measured current by the first
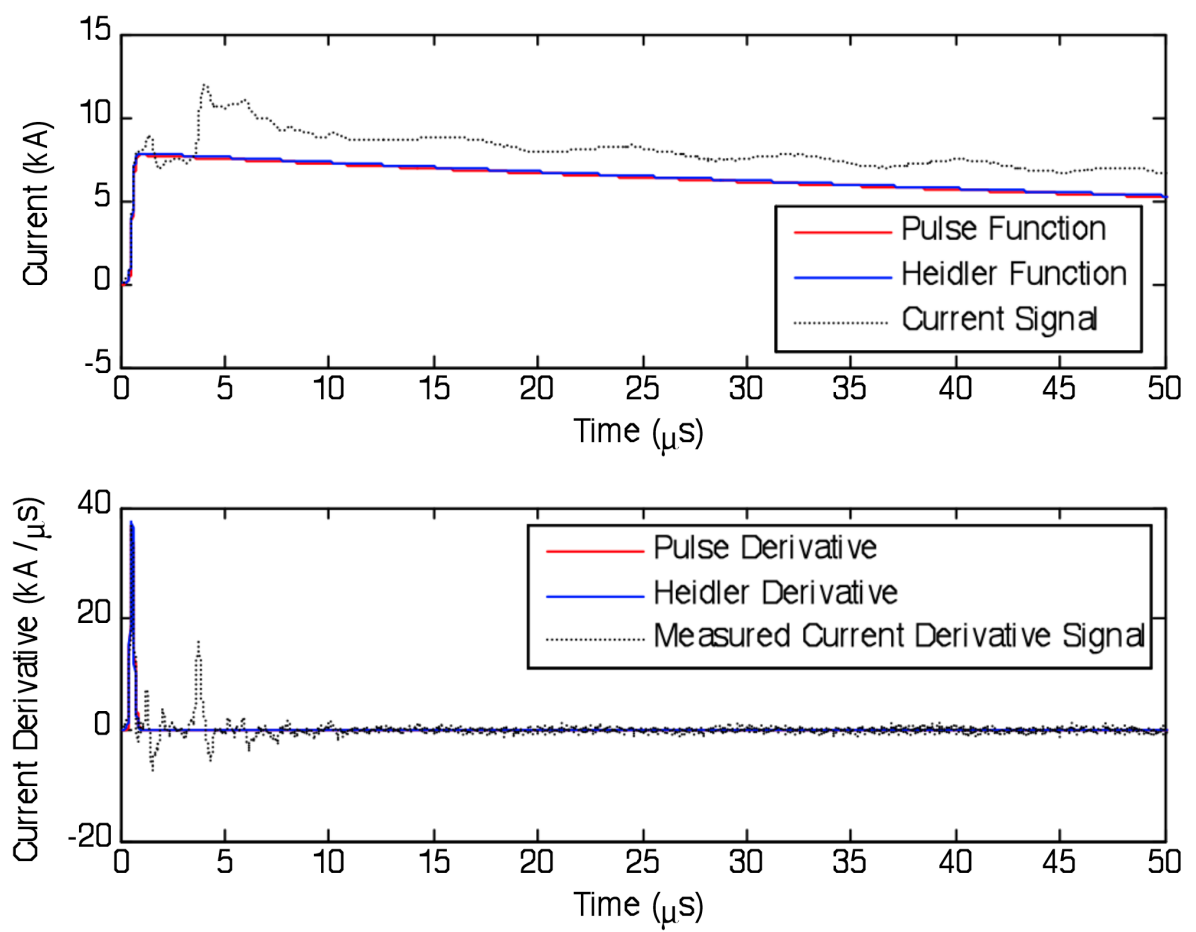

Fig. (3). Fitting results using the Pulse and Heidler functions for simulating the measured current derivative.
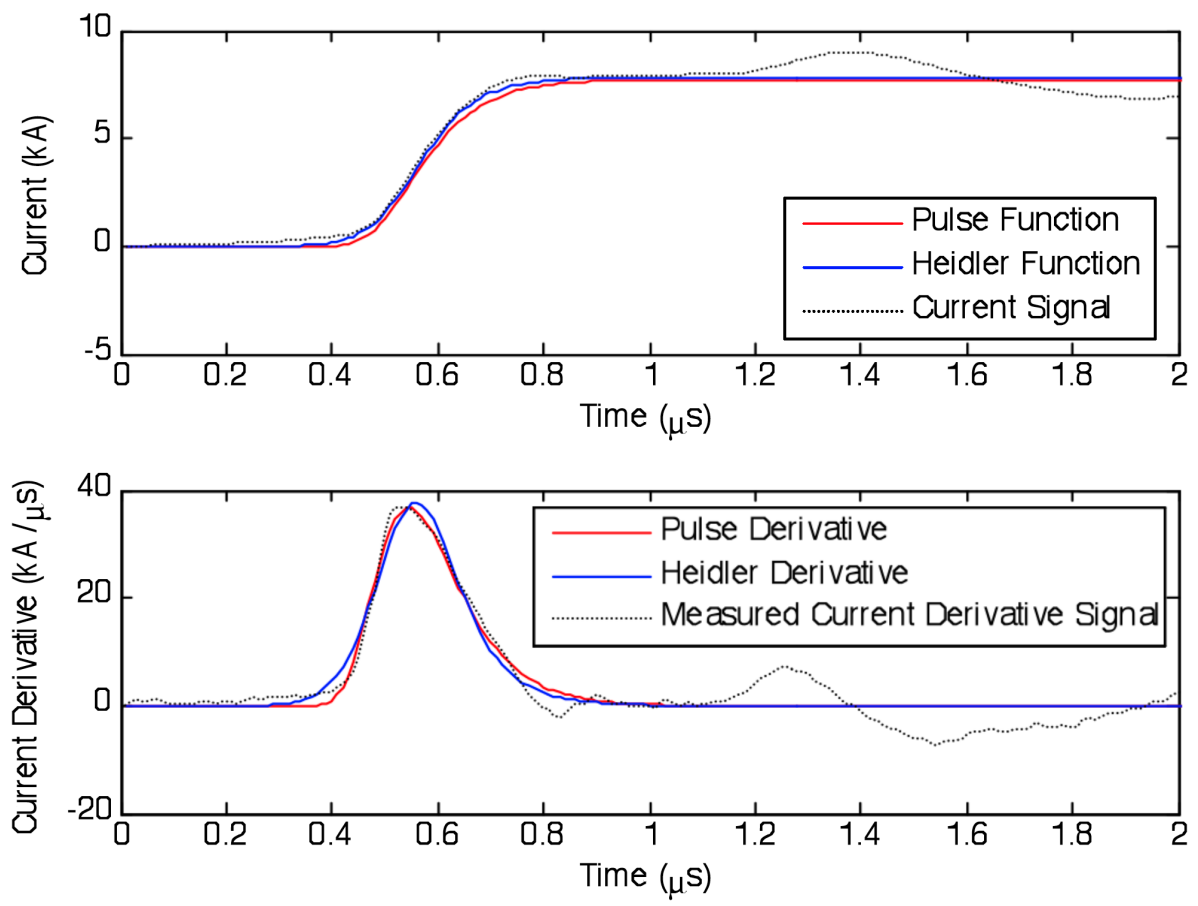

Fig. (4). Zoomed fitting results using the Pulse and Heidler functions for simulating the measured current derivative. 
estimating the decay time parameter before the application of the curve-fitting technique (see Figs. 1, 3). Furthermore, a general comparison, within the first time-window, between the simulated current, using the new technique (Fig. 4), and that using the ordinary technique (Fig. 2), reveals a substantial improvement in the simulation. Similarly, the simulated current derivative using the new technique is markedly improved (Figs. 2, 4).

In case of the Pulse function, the current derivative fitting is achieved while the corresponding current experienced intolerable error within the maximum current and its time of occurrence. For Heidler function, although the current derivative fitting is not as good as obtained in case of the Pulse function but the corresponding current is much better than that of the Pulse function, however, the maximum current and its time of occurrence were not achieved as well.

Since the maximum current value is a common problem it can be adjusted by forcing the maximum current $I_{\max }$ to have a certain value. This value can be obtained by visual investigation of the integration of the measured current derivative signal. By assigning the value of $I_{\max }$, the number of analytical parameters will be reduced to two, namely, $n$ and $\tau_{1}$.

As shown in Fig. 5, the Pulse function current signal is improved while the maximum steepness value, the time of occurrence of the maximum steepness and the zero crossing of the current derivative need to be improved to best fit the measured signal. Heidler function will have the same problems encountered using the Pulse function.

\subsection{Constraints}

Another way for improving the simulation is by introducing a constraint to force the analytical parameters to reach their optimal values. This constraint is applied by adjusting either the time of occurrence of the zero crossing of the current derivative or the time of occurrence of the maximum steepness of the current to a known value. We refer to these constraints in the paper as time-forcing constraints.

Applying this constraint using the known values of $\tau_{2}$ and $I_{\max }$, which were previously determined, $\tau_{1}$ can be expressed in terms of $n$ or vice versa. Accordingly, the number of analytical parameters of the functions will be reduced to one.

\subsubsection{Zero Crossing}

Firstly, the time forcing constraint will be applied to adjust the zero crossing of the current derivative to its measured value. In the case of the Pulse function, applying the constraint using equation (6), $n$ can be expressed in terms of $\tau_{1}$. By substituting $n$ in the Pulse function equation (3) and its derivative, equation (5), the only unknown parameter will be $\tau_{1}$. The function and its derivative can be reformatted as follows:

$$
\begin{aligned}
& I(t)=\frac{I_{\max }}{\eta} e^{-\frac{t}{\tau_{2}}}\left(1-e^{-\frac{t}{\tau_{1}}}\right)^{\frac{\tau_{1}}{\tau_{2}}\left(e^{\frac{t_{\max }}{\tau_{1}}}-1\right)} \\
& \frac{d I(t)}{d t}=\frac{I_{\max }}{\eta} e^{-\frac{t}{\tau_{2}}}\left(1-e^{-\frac{t}{\tau_{1}}}\right)^{\left.\frac{\tau_{1}}{\tau_{2}} e^{\left(\frac{t_{\max }}{\tau_{1}}\right.}-1\right)} *\left[\frac{\left(e^{\frac{t_{\max }}{\tau_{1}}}-1\right) e^{-\frac{t}{\tau_{1}}}}{\tau_{2}\left(1-e^{-\frac{t}{\tau_{1}}}\right)}-\frac{1}{\tau_{2}}\right]
\end{aligned}
$$

In the case of Heidler function, applying the constraint using equation (14), $\tau_{1}$ can be expressed in terms of $n$. By
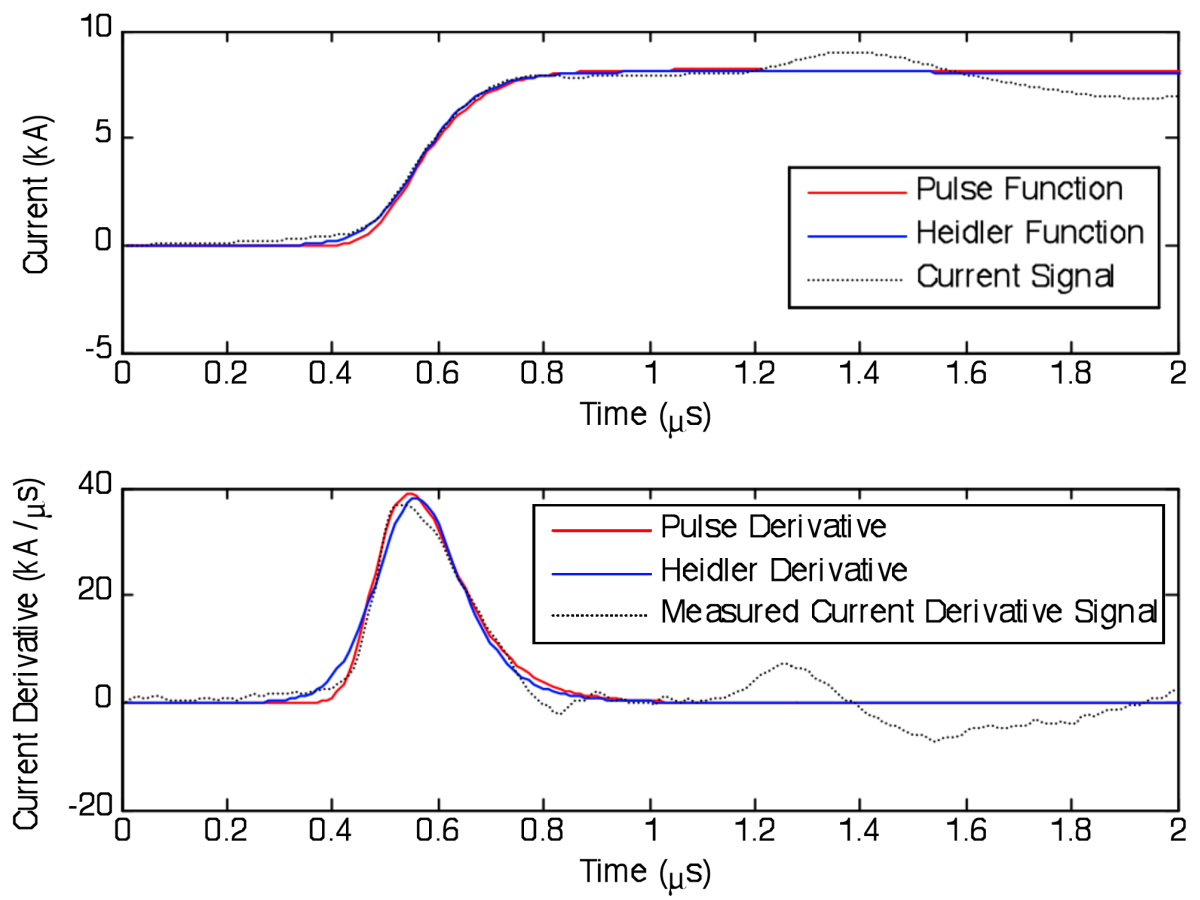

Fig. (5). Fitting result using the Pulse and Heidler functions for the measured current derivative with fixed $\tau_{2}$ and $I_{\max }$. 
substituting $n$ in Heidler function, equation (10), and its derivative, equation (12), the only unknown parameter will be $n$. The function and its derivative can be reformatted as follows:

$$
\begin{aligned}
& I(t)=I_{\max } \frac{t^{n}\left(1+\left(\frac{t_{\text {max }}}{t_{m s}}\right)^{n} \frac{n-1}{n+1}\right) e^{-\frac{t-t_{\max }}{\tau_{2}}}}{t_{\max }^{n}\left(1+\left(\frac{t}{t_{m s}}\right)^{n} \frac{n-1}{n+1}\right)} \\
& \frac{d I(t)}{d t}=I_{\max } \frac{t^{n}\left(1+\left(\frac{t_{\max }}{t_{m s}}\right)^{n} \frac{n-1}{n+1}\right) e^{-\frac{t-t_{\max }}{\tau_{2}}}}{t_{\max }^{n}\left(1+\left(\frac{t}{t_{m s}}\right)^{n} \frac{n-1}{n+1}\right)} * \\
& \left.\frac{n}{t}-\frac{1}{\tau_{2}}-\frac{n t}{\left(t_{m s}^{n} \frac{n+1}{n-1}\right)+t^{n}}\right]
\end{aligned}
$$

Fig. (6) shows that an overshoot for the maximum steepness value of the current signal occurred leading to a very fast current rise. Consequently, the zero crossing constraint was not able to achieve the optimal value of the analytical parameters.

\subsubsection{Maximum Steepness}

Secondly, the time forcing constraint will be applied to adjust the maximum steepness of the current signal to its measured value. In the case of the Pulse function, applying the constraint using equation (9), $n$ can be expressed in terms of $\tau_{1}$. By substituting $n$ in the Pulse function, equation (3), and its derivative, equation (5), the only unknown parameter will be $\tau_{1}$. The function and its derivative can be reformatted as follows:

$$
\begin{aligned}
& I(t)=\frac{I_{\max }}{\eta}\left(1-e^{-\frac{t}{\tau_{1}}}\right)^{\frac{t_{m s}}{\tau_{1}}} e^{-\frac{t}{\tau_{2}}} \\
& \frac{d I(t)}{d t}=\frac{I_{\max }}{\eta}\left(1-e^{-\frac{t}{\tau_{1}}}\right)^{\frac{t_{m s}}{\tau_{1}}} *\left[\frac{e^{\frac{t_{m s}-t}{\tau_{1}}}}{\tau_{1}\left(1-e^{-\frac{t}{\tau_{1}}}\right)}-\frac{1}{\tau_{2}}\right] e^{-\frac{t}{\tau_{2}}}
\end{aligned}
$$

Similarly, in the case of Heidler function, applying the constraint using equation (17), $\tau_{1}$ can be expressed in terms of $n$. By substituting $n$ in Heidler function, equation (10), and its derivative, equation (12), the only unknown parameter will be $n$. The function and its derivative can be reformatted as follows:

$$
\begin{aligned}
& I(t)=\frac{I_{\max }}{\eta} \frac{\left(\frac{t}{t_{m s}}\right)^{n} \frac{n-1}{n+1}}{1+\left(\frac{t}{t_{m s}}\right)^{n} \frac{n-1}{n+1}} e^{-\frac{t}{\tau_{2}}} \\
& \frac{d I(t)}{d t}=\frac{I_{\max }}{\eta} \frac{\left(\frac{t}{t_{m s}}\right)^{n} \frac{n-1}{n+1} e^{-\frac{t}{\tau_{2}}}}{1+\left(\frac{t}{t_{m s}}\right)^{n} \frac{n-1}{n+1}} *\left[\frac{n}{t}-\frac{1}{\tau_{2}}-\frac{n t^{n-1}}{\left(t_{m s}^{n} \frac{n+1}{n-1}+t^{n}\right)}\right]
\end{aligned}
$$
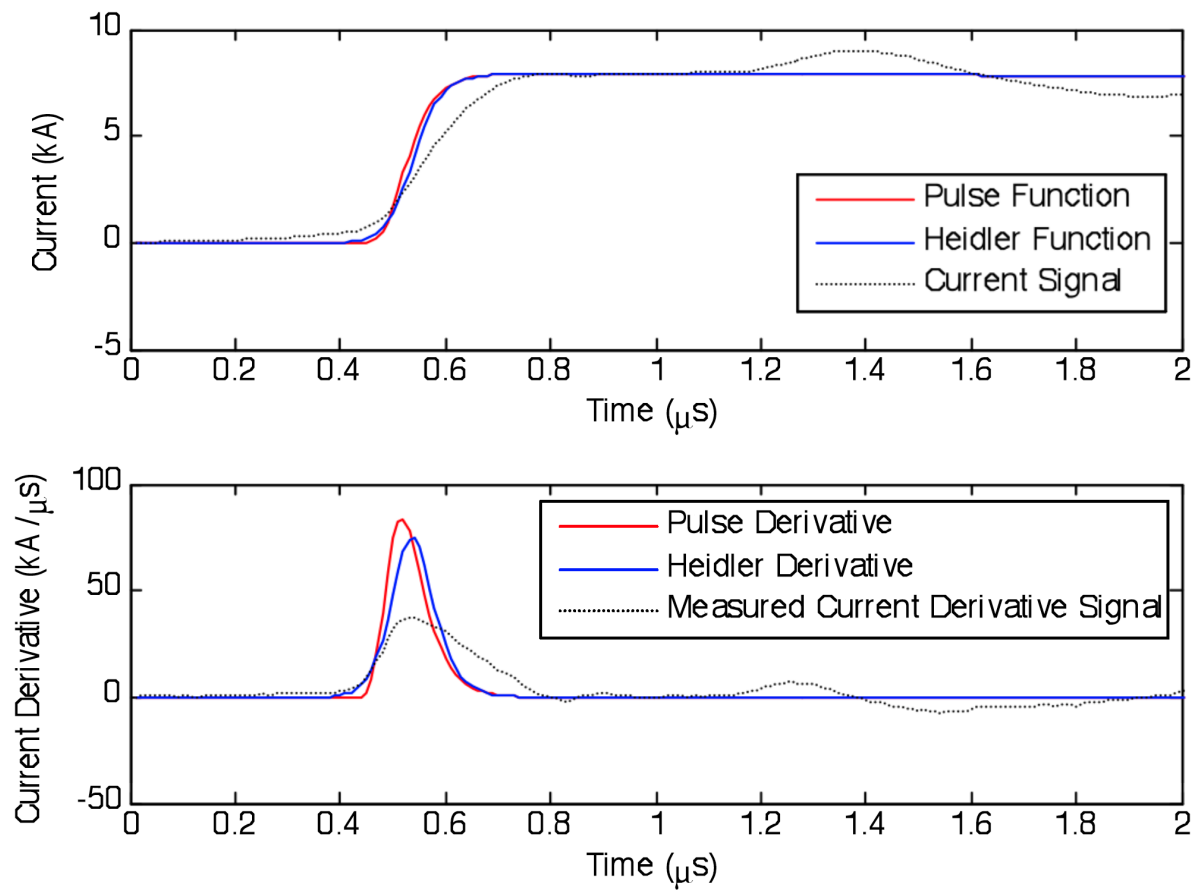

Fig. (6). Zoomed fitting results using the Pulse and Heidler functions for simulating the measured current derivative with fixed $\tau_{2}$ and $\mathrm{I}_{\mathrm{max}}$ and with forcing the time occurence of the zero crossing of the current derivative signal. 

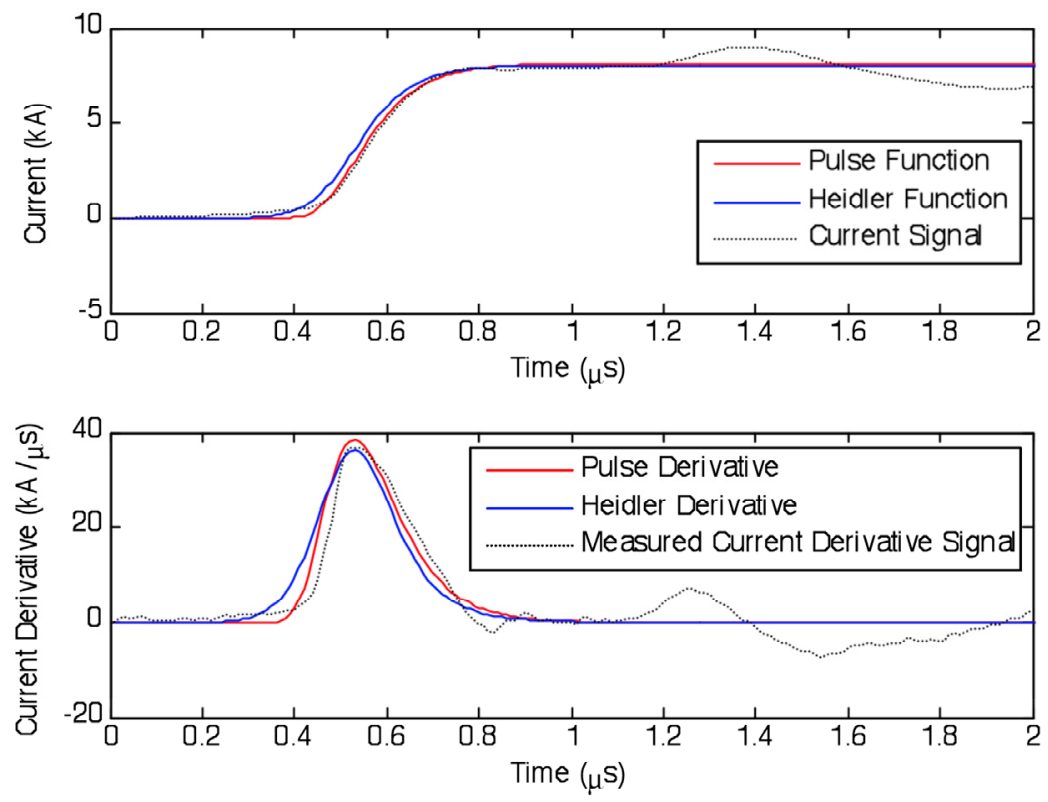

Fig. (7). Zoomed fitting results using the Pulse and Heidler functions for simulating the measured current derivative with fixed $\tau_{2}$ and $\mathrm{I}_{\max }$ and with forcing the time of occurrence of the maximum steepness of current signal.

Forcing the constraint regarding the time occurrence of the maximum steepness achieves the best fit for both simulating functions as shown in Fig. (7).

The descried fitting process represents the best method to reach the optimal value for simulating the measured signal. Not only fitting the full measured signal including the decay part but also calculating all current waveform parameters can be achieved. Table 1 compares the current waveform parameters extracted manually from the measured signal and the ones calculated using the this fitting process for the pulse function. Using this fitting process for the Pulse function all current waveform parameters can be calculated and full statistics of this parameters can be built through which lightning protection can be done.

Table 1. Comparison Between Measured and Calculated Current Waveform Parameters

\begin{tabular}{|c|c|c|}
\hline & $\begin{array}{c}\text { Measured } \\
\text { Signal }\end{array}$ & $\begin{array}{c}\text { Pulse } \\
\text { Function }\end{array}$ \\
\hline \hline Maximum Current $(\mathrm{kA})$ & 7906.5 & 7906.5 \\
\hline Maximum Steepness $(\mathrm{kA} / \mu \mathrm{s})$ & 36.98 & 38.5735 \\
\hline Rise Time $(\mu \mathrm{s})$ & 0.25 & 0.2343 \\
\hline Decay Time $(\mu \mathrm{s})$ & ------- & 285.97 \\
\hline Charge Transferred $(\mathrm{C})$ & ------- & 0.9393 \\
\hline Waveform width half of maximum current $(\mu \mathrm{s})$ & -------- & 89.204 \\
\hline
\end{tabular}

It is very important to clarify that the better accuracy and the easiness achieved when using the simulated function to get the value of the current wave form parameters than using the traditional manual methods. Using the simulating function gave more freedom for calculating other parameters that was difficult to measure using the manual methods such as the decay time, charge being transferred and the wave form width for half maximum current.

\section{CUMULATIVE STATISTICAL DISTRIBUTION}

The described fitting process is applied on 15 flashes, containing 31 return strokes. The calculated current waveform parameters were used to form statistics to determine the probability distribution of the value of each parameter, including the range and the $50 \%$ probability level, which is fundamental in building lightning protection systems.

\subsection{Current Peak}

The cumulative distribution of the current peak of the lightning return-stroke current for the analyzed signals is shown in Fig. (8). The current peak, determined from the simulated function, varies from a minimum value of $2.59 \mathrm{kA}$ to a maximum value of $11.08 \mathrm{kA}$. The average value of the current peak was found to be $5.57 \mathrm{kA}$. It was observed that in $50 \%$ of the recorded lightning strokes, the current peak exceeded $5.73 \mathrm{kA}$, in $5 \%$ of recorded signals, the current peak exceeded $10.21 \mathrm{kA}$ and in $95 \%$ of captured strokes the current peak exceeded $2.98 \mathrm{kA}$.

\subsection{Maximum Steepness}

For the maximum steepness, its cumulative distribution is shown in Fig. (9). The lightning signals analyzed had a minimum value of $11.65 \mathrm{kA} / \mu \mathrm{s}$ and maximum value of $54.68 \mathrm{kA} / \mu \mathrm{s}$ for the maximum steepness. The average value for the maximum steepness was found to be $31.33 \mathrm{kA} / \mu \mathrm{s}$. In $95 \%$ of the signals, the maximum steepness came to be higher than $14.31 \mathrm{kA} / \mu \mathrm{s}$ and in $5 \%$ of the cases the maximum steepness was higher than $51.21 \mathrm{kA} / \mu \mathrm{s}$. The $50 \%$ probability value for the maximum steepness was found to be $34.31 \mathrm{kA} / \mu \mathrm{s}$.

\subsection{Rise Time to the Current Peak}

The $10 \%$ to $90 \%$ risetime to the current peak for each of the analyzed signals was determined from the simulated 


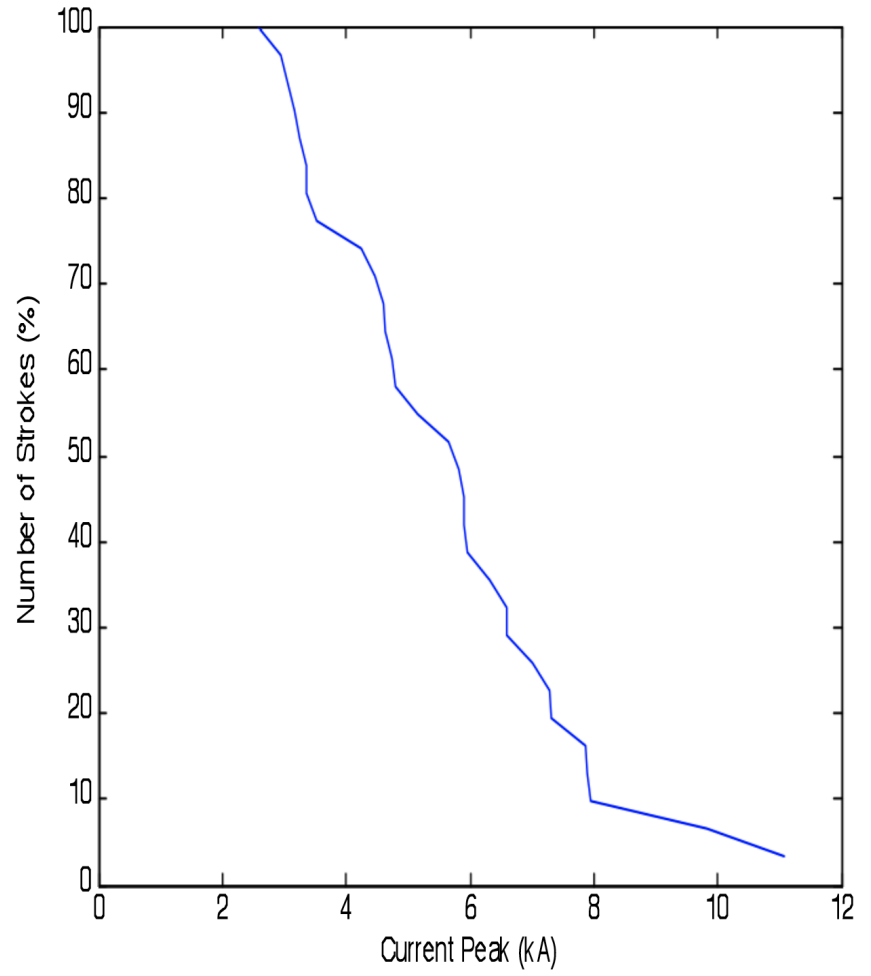

Fig. (8). Cumulative distribution of the current peak.

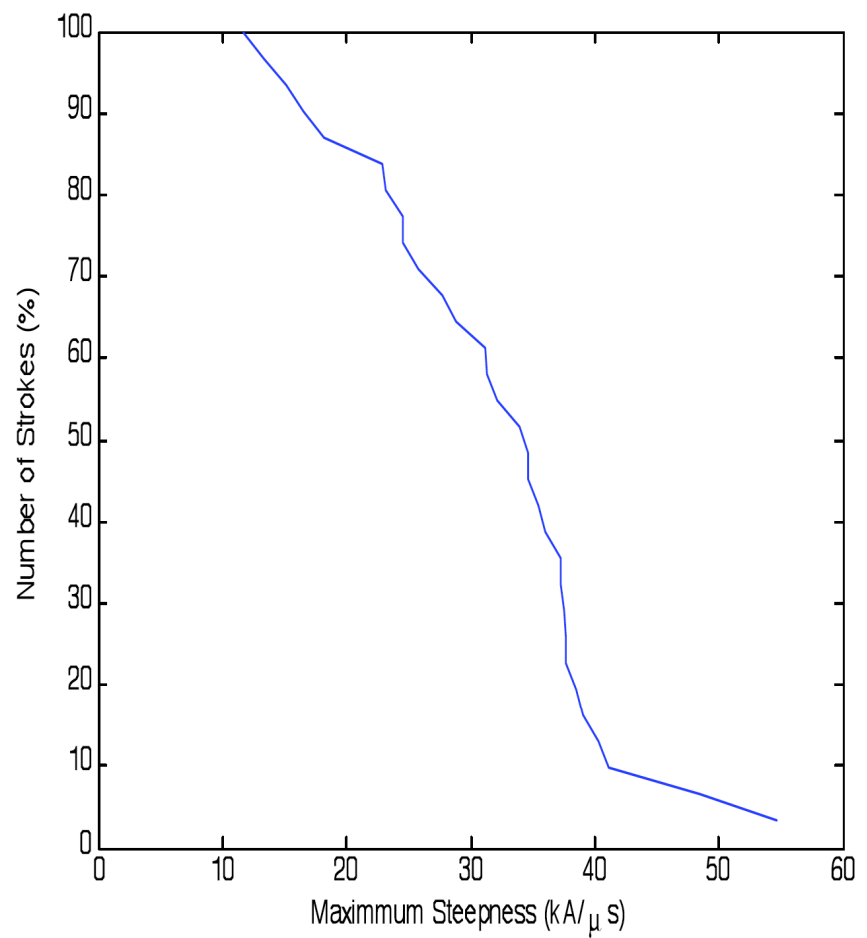

Fig. (9). Cumulative distribution of the maximum steepness.

function. Fig. (10) shows the cumulative distribution of the current risetime. The minimum recorded risetime to the current peak was 124 ns while the maximum recorded value was 524 ns with an average of 242.3 ns. It is important realize this large range of variation in the current risetime when designing lightning protection systems. For the analyzed signals, the current risetime exceeds $143 \mathrm{~ns}, 211 \mathrm{~ns}$ and $481 \mathrm{~ns}$ in $95 \%, 50 \%$ and $5 \%$ of the cases, respectively.

\subsection{Decay Time from the Current Peak}

The decay time of the current peak is an important waveform parameter. The $90 \%$ to $10 \%$ current decay time from the current peak for each of the analyzed signals was determined. The cumulative distribution of the current decay time is shown in Fig. (11). The minimum value of the current decay time was found to be $65.88 \mu \mathrm{s}$ with maximum value of $537.13 \mu \mathrm{s}$. The average value of the current decay time is $255.64 \mu \mathrm{s}$. In $95 \%$ of the cases, the current decay time exceeds $75.26 \mu \mathrm{s}$ and in $5 \%$ of the cases, it exceeds $523.6 \mu \mathrm{s}$. The $50 \%$ probability value of the current decay time is $261.3 \mu \mathrm{s}$.

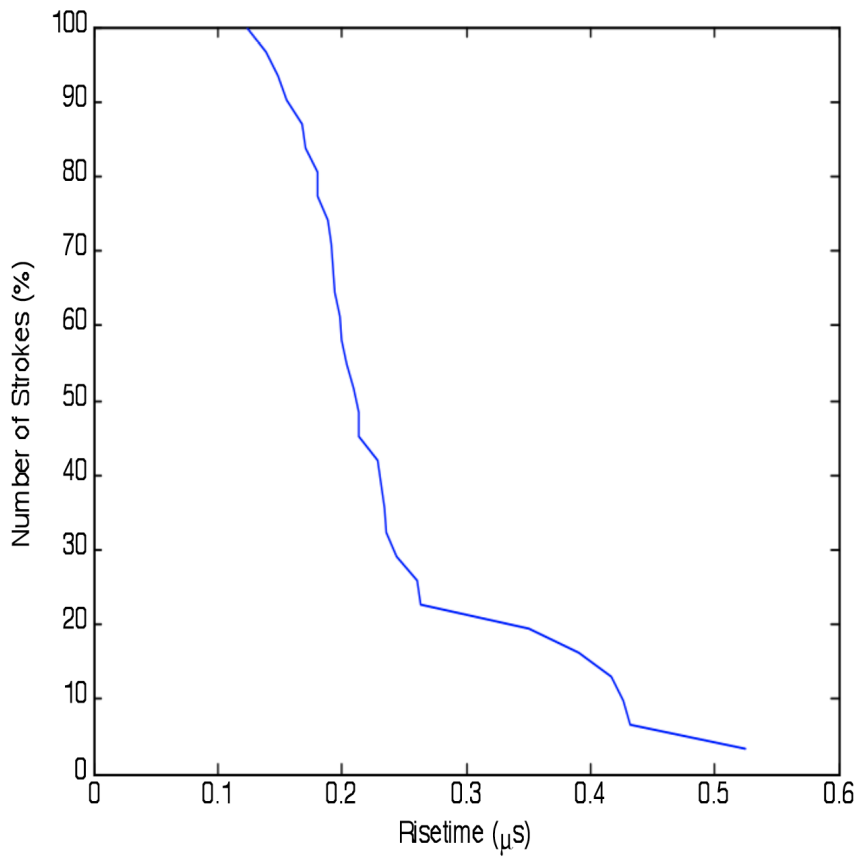

Fig. (10). Cumulative distribution of the risetime to the current peak.

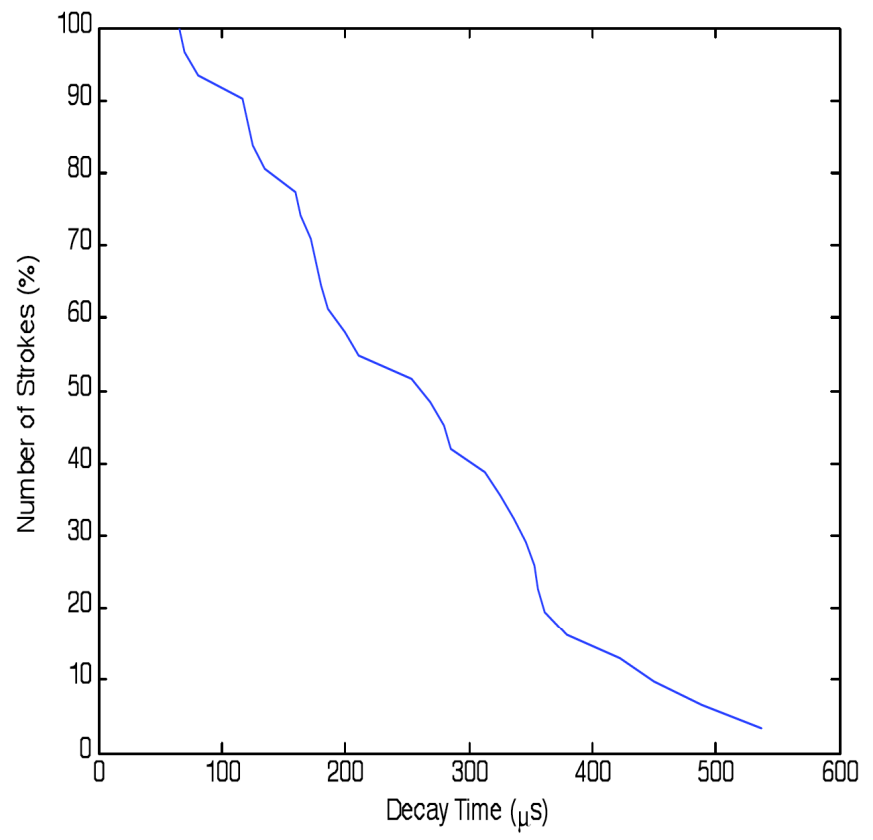

Fig. (11). Cumulative distribution of the decay time from the current peak. 


\subsection{Current Pulse Width}

The current pulse width at the half current peak level for each of the analyzed signals was calculated by determining the time difference between the time at the $50 \%$ level of current peak at the decay side of the current pulse and the $50 \%$ level of the current peak at the rising side of the current pulse. Fig. (12) shows the cumulative distribution of the current pulse width. The minimum recorded current pulse width is $21.47 \mu \mathrm{s}$ and the maximum recorded value is 157.51 $\mu \mathrm{s}$, with an average of $83.99 \mu \mathrm{s}$. The large range of variation in current pulse width is important to take into consideration by engineers working on lightning protection methods. For the analyzed signals, the current pulse width $75.23 \mu \mathrm{s}, 261.3$ $\mu \mathrm{s}$ and $512.3 \mu \mathrm{s}$ in $95 \%, 50 \%$ and $5 \%$ of the cases, respectively.

\subsection{Charge}

The charge is one of the most important waveform parameters in the area of protection. The charge for each of the analyzed signals was obtained by integrating the current. The cumulative distribution of the charge is shown in Fig. (13). The minimum value of the charge is $0.1187 \mathrm{C}$, while the maximum value is $1.897 \mathrm{C}$. The average value of the charge is $0.6642 \mathrm{C}$. In $95 \%$ of the cases, the charge exceeds $0.154 \mathrm{C}$ and in $5 \%$ of the cases, the charge exceeds $1.671 \mathrm{C}$. In $50 \%$ of the analyzed signals, the charge exceeds $0.599 \mathrm{C}$.

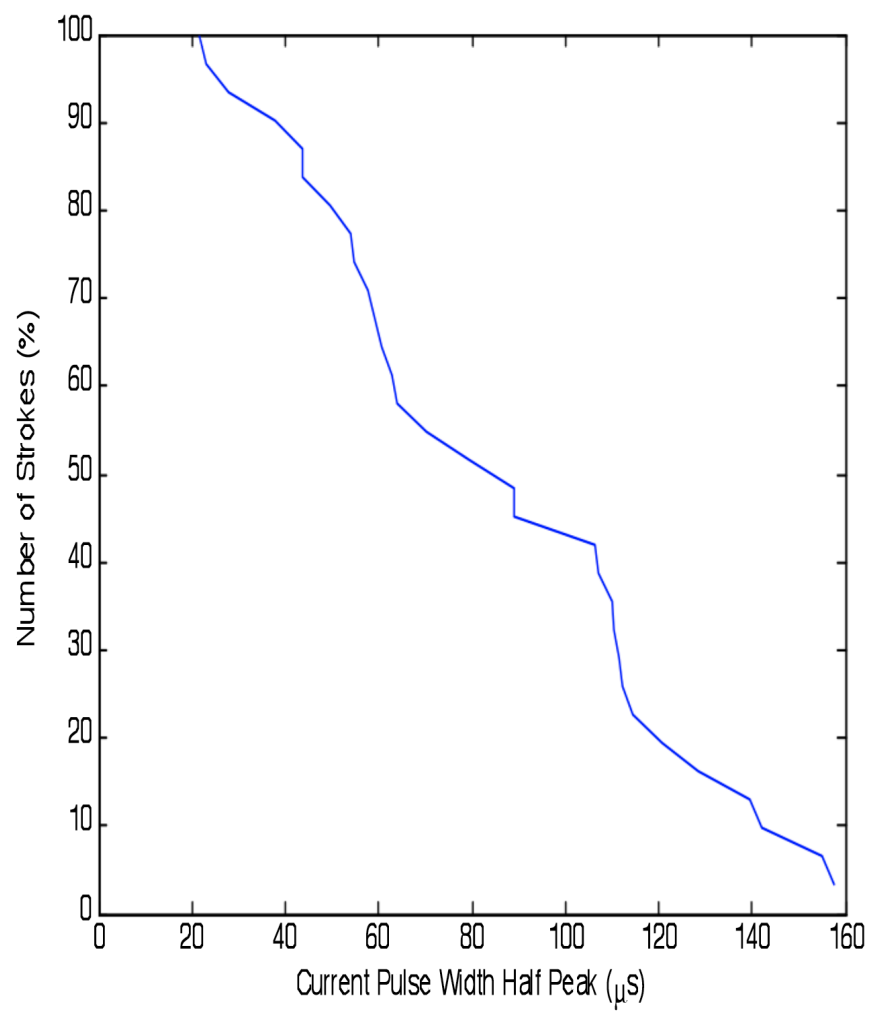

Fig. (12). Cumulative distribution of the current pulse width.

Table 2 summarizes the cumulative statistics of current waveform parameters, including each waveform parameter value at $95 \%, 50 \%$ and $5 \%$ of the cases. Also, it includes the maximum value, the minimum value, and the average value of each waveform parameters.

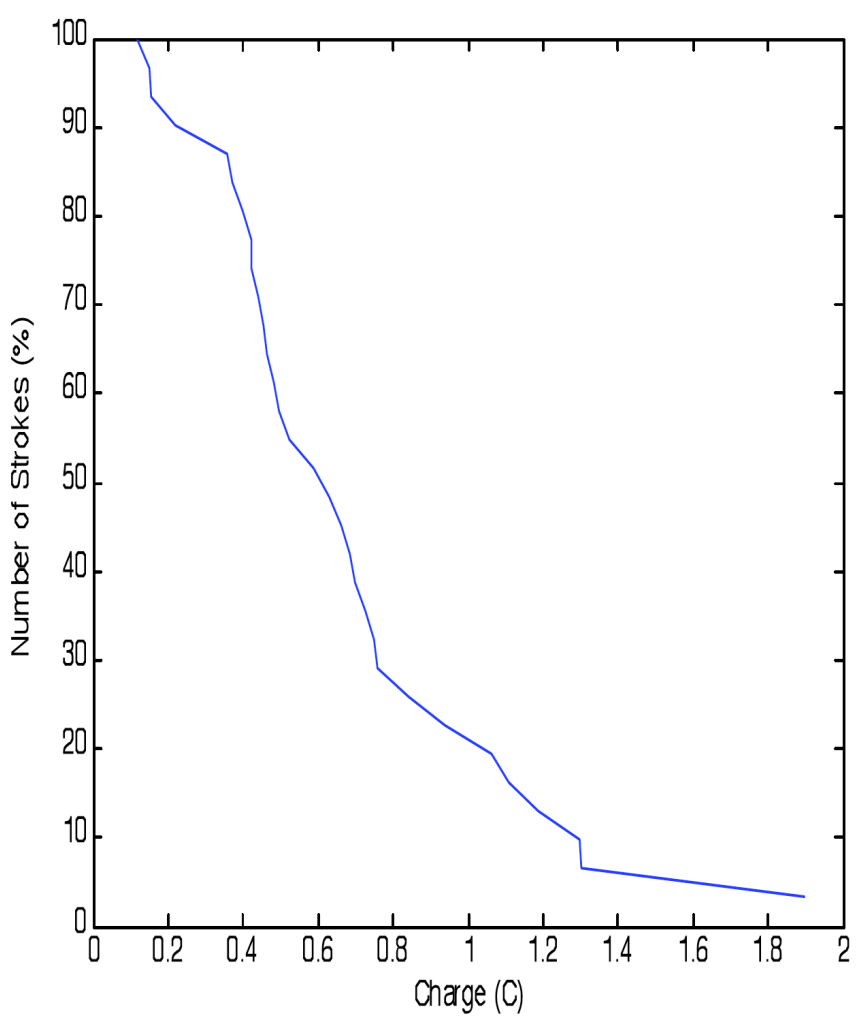

Fig. (13). Cumulative distribution of the charge.

\section{CONCLUSIONS}

From the previous results, it can be generally concluded that the simulation of the current derivative yields closer fit to the measurement, when compared with the fit resulting from simulating the current. Using artificial signals, which are free of noise and reflections, proved to be very efficient in the determination of initial value estimation.

The decoupling behaviour of the Pulse and Heidler functions helped in dividing the signal into three parts and avoiding the use of the second part, which is highly distorted by reflections, in the fitting process. The third part was used for estimating the decay parameter $\tau_{2}$. The first part (mainly the rise portion of the signal) was used to estimate the other parameters. Using the approximation of the decaying part for the simulating functions lead to same value of $\tau_{2}$ and $\mathrm{I}_{\max }$ for both functions.

Finally, using the time-forcing constraint for the maximum steepness of the current for fitting the rising part of the signal, given $\tau_{2}$ and $I_{\max }$, reduced the fitting process to a single-parameter estimation, and allowed the comparison between the Pulse function and Heidler function for simulating the measured. Although Heidler function is widely used in fitting the return-stroke current signal, the Pulse function proved to be a better fit for the lightning return-stroke current, measured at the $\mathrm{CN}$ Tower. The Pulse function is used to calculate the waveform parameters that were manually determined. Also, other important parameters that could not be determined previously were systematically computed. Newly determined parameters are the pulse width at half peak level, decay time, charge being transferred. 
Table 2. Summary of the Cumulative Statistics of the Current Waveform Parameters

\begin{tabular}{|l|c|c|c|c|c|c|}
\hline \multicolumn{1}{|c|}{ Waveform Parameter } & Minimum & Maximum & Average & $\mathbf{5 \%}$ & $\mathbf{5 0 \%}$ & $\mathbf{9 5 \%}$ \\
\hline \hline Current Peak $(\mathrm{kA})$ & 2.895 & 11.08 & 5.572 & 10.21 & 5.73 & 2.98 \\
\hline Maximum Steepness $(\mathrm{kA} / \mu \mathrm{s})$ & 11.65 & 54.68 & 31.3261 & 51.21 & 34.31 & 14.31 \\
\hline Risetime to Current Peak $(\mu \mathrm{s})$ & 0.124 & 0.524 & 0.2423 & 0.481 & 0.211 & 0.143 \\
\hline Decay Time from current peak $(\mu \mathrm{s})$ & 65.88 & 537.13 & 255.64 & 523.6 & 261.3 & 75.26 \\
\hline Current Pulse Width $(\mu \mathrm{s})$ & 21.47 & 157.51 & 83.99 & 512.3 & 261.3 & 75.23 \\
\hline Charge $(\mathrm{C})$ & 0.1187 & 1.897 & 0.6642 & 1.671 & 0.5986 & 0.1542 \\
\hline
\end{tabular}

\section{ACKNOWLEDGEMENT}

Declared none.

\section{CONFLICT OF INTEREST}

The authors confirm that this article content has no conflicts of interest.

\section{REFERENCES}

[1] Rachidi F, Janischewskyj W, Hussein AM, et al. Current and electromagnetic field associated with lightning return-stroke to tall towers. IEEE Trans Electromagn Compatibility 2001; 43(3): 35667.

[2] Rakov VA, Uman MA. Review and evaluation of lightning return stroke models including some aspects of their application. IEEE Trans Electromagn Compatibility 1998; 40(4): 403-26.

[3] Feizhou Z, Shanghe L. A new function to represent the lightning return-stroke current. IEEE Trans Electromagn Compatibility 2002; 44(4): 595-7.

[4] Milewski M, Hussein AM. Tall-structure lightning return-stroke modelling. Proceeding of the $14^{\text {th }}$ International Middle East Power System Conference; Dec 19-21; Cairo University, Cairo: Egypt 2010; pp. 947-52.

[5] Milewski M, Hussein AM. Lightning return-stroke transmission line model based on $\mathrm{CN}$ Tower lightning data and derivative of Heidler function. Canadian Conference on Electrical and Computer
Engineering, The Wonders of Technology; May 2008; vol. 11, no. 2. Niagara Falls, Ontario: Canada; 2008; pp. 141-50.

[6] Bitner K, Hussein AM. Modelling of the CN Tower lightning return-stroke current derivative. Proceedings of the $28^{\text {th }}$ International Conference on Lightning Protection; 2006 September 18-22; Kanazawa: Japan; 2006; pp. 261-6.

[7] Jones RD. On the use of tailored return-stroke current representation to simplify the analysis of lightning effect on systems. IEEE Trans Electromagn Compatibility 1977; 19(2): 95-6.

[8] Gardner RL, Baker L, Baum CE, Andersh DJ. Comparison of lightning with public domain HEMP waveforms on the surface of an aircraft. $6^{\text {th }}$ EMC Symposium; Zurich 1985; pp. 175-80.

[9] Yazhou C, Shanghe L, Xiaorong W, Feizhou Z. A new kind of channel-base current function. Proceedings of the $3^{\text {rd }}$ International Symposium on Electromagn Compatibility; May 2002; pp. 304-7.

[10] Heidler F, Cvetic JM, Stanic BV. Calculation of lightning current parameters. IEEE Trans Power Deliv 1999; 14(2): 399-404.

[11] Heidler F, Cvetic JM. A class of analytical functions to study the lightning effects associated with the current front. Eur Trans Electr Power 2002; 12(2): 141-50.

[12] Elrodesly K. Comparison between Heidler Function and the Pulse Function for Modeling the Lightning Return-Stroke Current. MASc Thesis. Toronto, Ontario, Canada: Ryerson University, July 2010.

[13] Nedjah O, Hussein AM, Krishnan S, Soludeh R. Comparative study adaptive de-noising techniques for lightning current derivative signals. Eur Assoc Signal Process J 2010; 20(2): 607-18.

(C) Elrodesly and Hussein; Licensee Bentham Open.

This is an open access article licensed under the terms of the Creative Commons Attribution Non-Commercial License (http://creativecommons.org/licenses/by-nc/3.0/) which permits unrestricted, non-commercial use, distribution and reproduction in any medium, provided the work is properly cited. 